To appear in Proceedings of the Dynamics Days Asia Pacific Conference, 13-16 July, 1999, Hong Kong (Physica A, 2000).

\title{
Modelling High-frequency Economic Time Series
}

\author{
Lei-Han Tang ${ }^{a}$ and Zhi-Feng Huang ${ }^{a, b}$ \\ ${ }^{a}$ Department of Physics and Center for Nonlinear Studies \\ Hong Kong Baptist University, Kowloon Tong, Hong Kong \\ ${ }^{b}$ Center for Advanced Study, Tsinghua University, Beijing 100084, P. R. China
}

\begin{abstract}
The minute-by-minute move of the Hang Seng Index (HSI) data over a four-year period is analysed and shown to possess similar statistical features as those of other markets. Based on a mathematical theorem [S. B. Pope and E. S. C. Ching, Phys. Fluids A 5, 1529 (1993)], we derive an analytic form for the probability distribution function (PDF) of index moves from fitted functional forms of certain conditional averages of the time series. Furthermore, following a recent work by Stolovitzky and Ching, we show that the observed PDF can be reproduced by a Langevin process with a move-dependent noise amplitude. The form of the Langevin equation can be determined directly from the market data.
\end{abstract}

The availability of high-frequency economic time series, with a sampling rate of every few seconds, has generated a great deal of theoretical interest in the econometrics and the econophysics community[1-4]. Attempts have been made to devise models which produce time series with similar statistical characteristics as those of real markets. Many of these studies are based on variants of the Autogressive Conditional Heteroskedasticity (ARCH) process first introduced by Engle[5] to analyze the quarterly consumer price index in the UK over the period 1958 to 1977, and the generalised ARCH (GARCH) process which offers a more flexible description of the volatility memory effect (i.e., lag structure)[6]. The nonlinearity in the regression models makes it possible to generate probability distribution functions (PDF) with fat tails, a characteristic of financial data first noted by Mandelbrot[7]. However, since all these processes are discrete in time, an immediate question to ask is whether the quality of the modelling depends on the time unit chosen and if there is a time scale which is the most natural of all. Indeed, when the time step is not chosen properly, one has to either introduce many terms in the regression expression

Preprint submitted to Elsevier Preprint 30 October 2018 


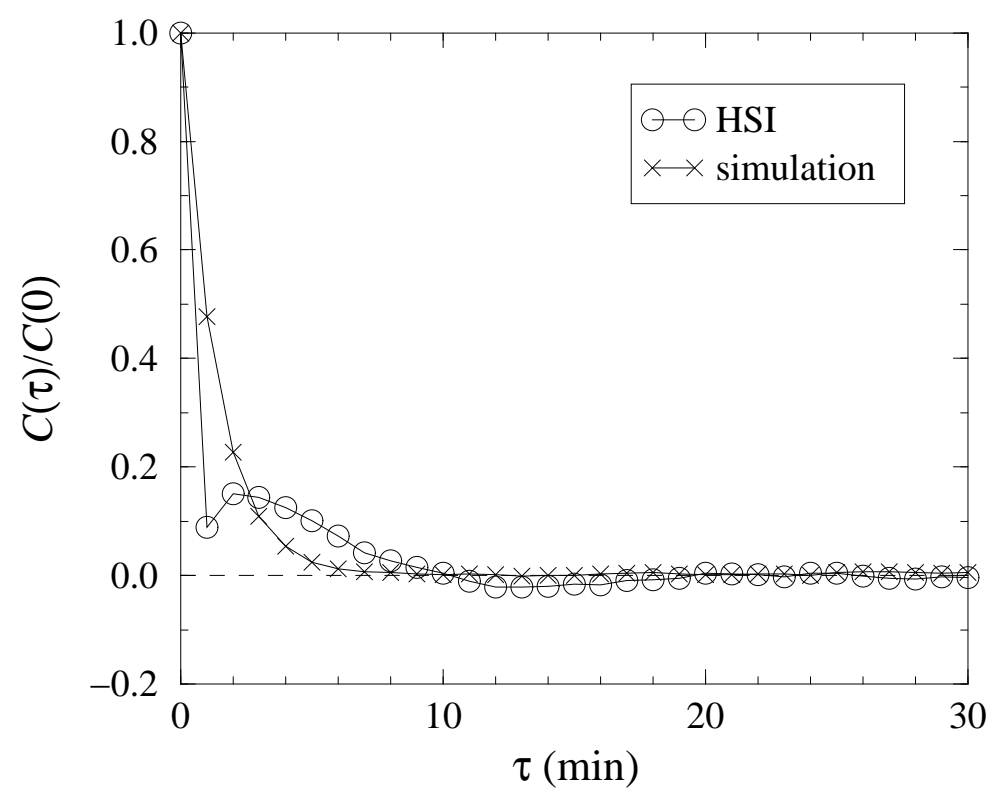

Fig. 1. Normalised linear two-point correlation function of the minute-by-minute HSI moves over the four-year period 1994 to 1997. Note the weak oscillations of the correlation function, indicating a slightly under-damped behaviour. Also shown is the same correlation function calculated from the simulation using a Langevin equation.

[the $\operatorname{GARCH}(p, q)$ model] to take into account memory effects, or to miss some of the important short-time statistics.

An alternative approach, which partially circumvents the above difficulty, is to model the market price move as a continuous time process. Continuous time stochastic processes are quite familiar to physicists, ranging from simple Brownian motion to the fully-developed turbulence. In fact, the high-frequency market price movements have much in common with the velocity or temperature time series in turbulent flows[8-11], an analogy we exploit in this paper. To put this statement on more quantitative terms, let us first summarise two salient statistical features which seem to be universally true for all major stock indices [4].

(i) Short linear correlation of price moves - For a given stock index $S(t)$, one may define the price move over a fixed time interval $\delta$ (say one minute),

$$
x(t)=S(t)-S(t-\delta)
$$

It has been shown that the "linear correlation"

$$
C(\tau)=\langle x(t+\tau) x(t)\rangle
$$




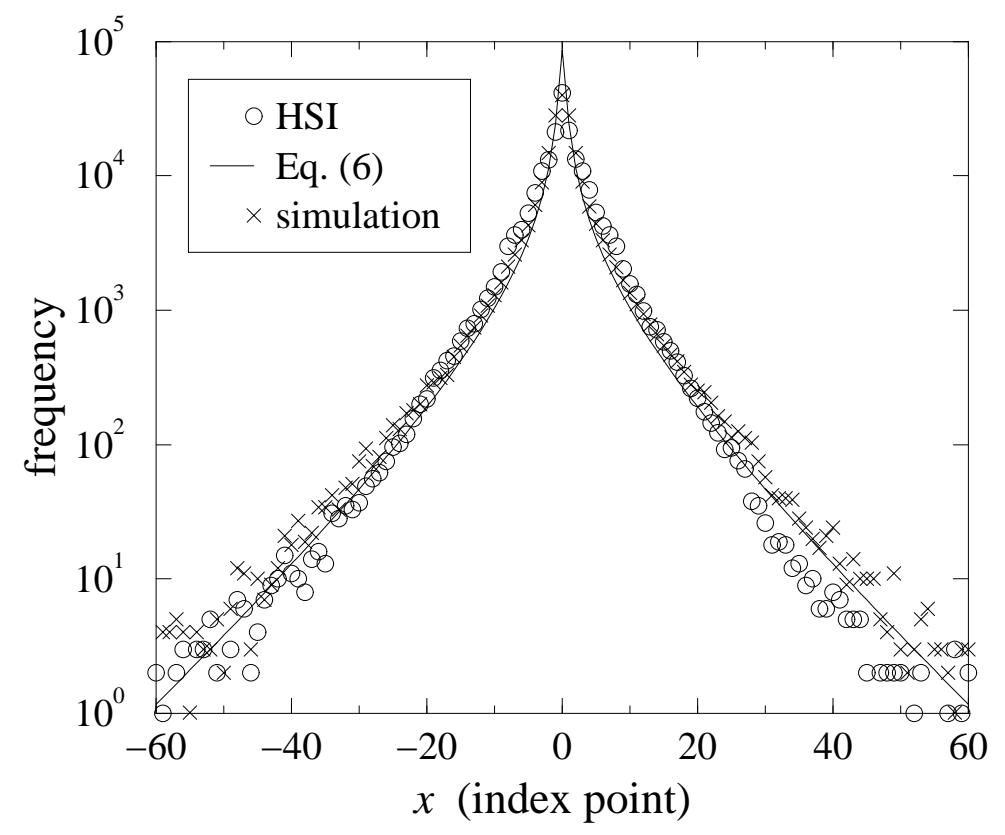

Fig. 2. The (unnormalised) probability distribution function (PDF) of the minute-by-minute HSI move $(N=201704$ events $)$. Data in the first twenty minutes of each morning trading session are discarded. Also shown are the PDF calculated from Eq. (6) by fitting the conditional averages (solid line), and the PDF of the time series from the simulation (crosses).

decays to zero very rapidly, on the order of ten minutes. We have analysed the minute-by-minute Hang Seng Index (HSI) data collected over a four year period January 1994 - December, 1997. Figure 1 shows the linear correlation function $C(\tau)$ with $\delta=1 \mathrm{~min}$. It is seen that $C(\tau)$ becomes nearly zero after a period of ten minutes or so. The decay is however not completely monotone, suggesting that the market is slightly under-damped.

(ii) Nongaussian distribution of price moves with fat tails — The fat tails of the PDF $P(x)$ of stock price moves are well-known and have also been observed for the movement of foreign currency exchange rates. Mandelbrot has observed that $P(x)$ often decays as a power-law function of $|x|$, and hence, combining with (i), the stock index can be considered as a realisation of Lévy walk[7]. From the analysis of the high-frequency S\&P 500 data, Mantegna and Stanley showed that a truncated Lévy distribution offers a better description of the PDF[2]. Figure 2 shows the PDF for the HSI minute-by-minute move data $x(t)$ (open circles) on a semi-log scale, collected over the same period as in Fig. 1. It is seen that the decay at large $|x|$ can be well-described by a simple exponential function, as observed previously in Ref. [2]. For small $|x|$, a different behaviour is seen. A noticeable feature is the cusp-like singularity at $x=0$, which is so far unexplained.

The peculiar form of the PDF as seen in Fig. 2 has in fact been observed in 

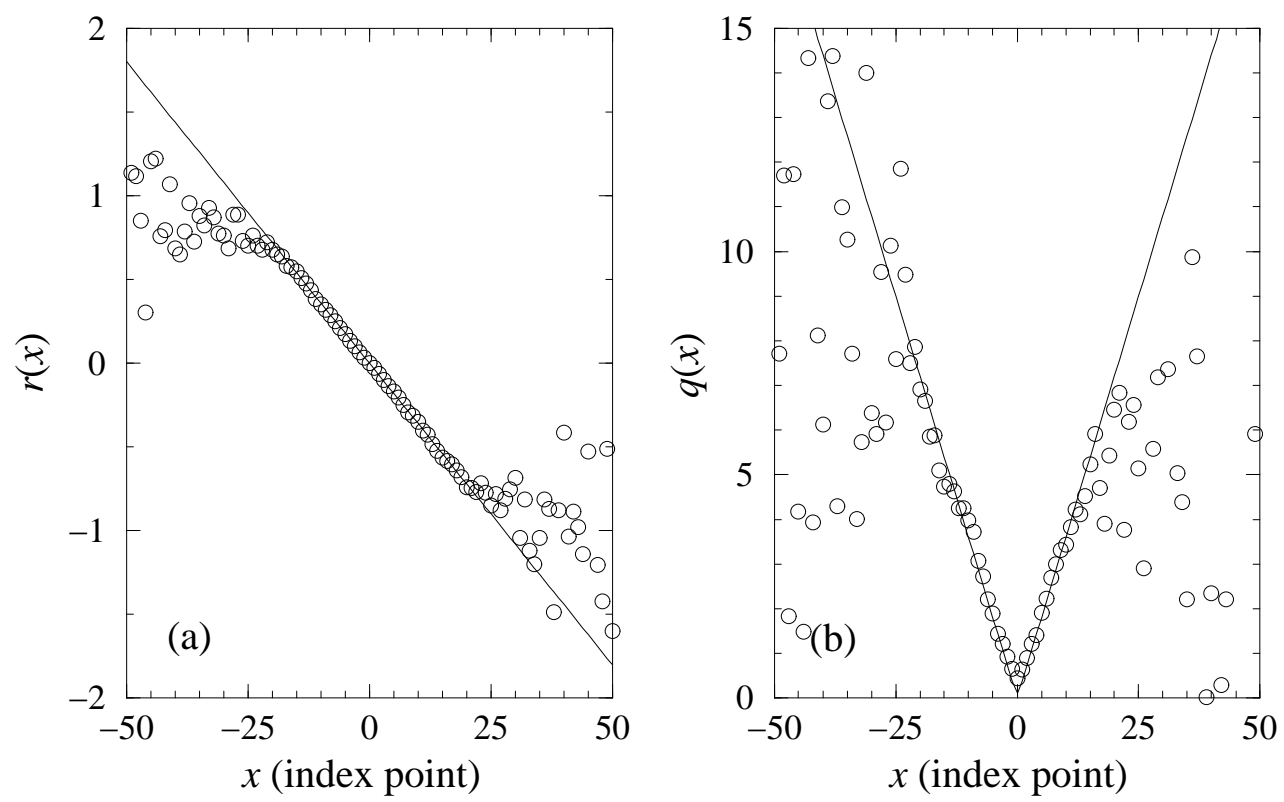

Fig. 3. (a) Conditional average $r(x)$ (circles) and the linear fit (solid line). (b) Conditional average $q(x)$ (circles) and a nearly linear fit (solid line).

a physical context[10]. In analysing the temperature time series of thermal convection in the "hard-turbulence" regime, Pope and Ching[11] considered the following conditional averages for a twice-differentiable time series $x(t)$,

$$
r(x)=\frac{\langle\ddot{x} \mid x\rangle}{\left\langle\dot{x}^{2}\right\rangle}, \quad q(x)=\frac{\left\langle\dot{x}^{2} \mid x\right\rangle}{\left\langle\dot{x}^{2}\right\rangle} .
$$

Here $\langle\cdot \mid x\rangle$ denotes the average of a given quantity over those data points in the time series where $x(t)=x$. From the stationarity of the PDF, they proved that the PDF and the conditional averages $r(x)$ and $q(x)$ are related through the following equation,

$$
P(x)=\frac{C}{q(x)} \exp \left[\int_{0}^{x} \frac{r\left(x^{\prime}\right)}{q\left(x^{\prime}\right)} d x^{\prime}\right] .
$$

Using the turbulent temperature time series data as input, they showed that $r(x)$ is generally linear in $x$ with a negative coefficient. In the soft turbulence regime, $q(x)$ is nearly constant. From Eq. (4), the resulting PDF is gaussian as observed. On the other hand, in the hard turbulence regime, $q(x)$ increases with increasing $|x|$, giving rise to fat tails in the PDF.

Figure 3 shows $r(x)$ and $q(x)$ computed using the HSI move time series. Indeed, the shape of these two functions are very much like the temperature data in Ref. [11], although there are small differences. The data can be fitted to the 
following functional forms,

$$
r(x)=-R x, \quad q(x)=Q\left(x^{2}+a^{2}\right)^{1 / 2},
$$

where $R=0.036$ and $Q=0.36$. The round-off parameter $a$ can not be determined precisely because of the discreteness of the index data. Instead, we fix its value from the normalisation condition. Substituting Eq. (5) into Eq. (4), and carrying out the integration, we obtain,

$$
P(x)=\frac{C^{\prime}}{\left(x^{2}+a^{2}\right)^{1 / 2}} \exp \left[-\alpha\left(x^{2}+a^{2}\right)^{1 / 2}\right]
$$

where $\alpha=R / Q=0.1$ and $C^{\prime-1}=2 K_{0}(\alpha a)$, with $K_{0}(u)$ being a modified Bessel function of the second kind. The solid line in Fig. 2 is produced by taking $a^{2}=0.1$. As can be seen, the agreement between the original data and the fitted form (6) is rather satisfactory.

It is worth noting that the functional form (6) we propose is quite different from those of earlier studies[12]. The behaviour of the PDF around $x=0$ is controlled by the parameter $a$. A sharp peak is produced when $a$ becomes very small. In this respect, $a$ serves the purpose of a cut-off related to the discreteness of the underlying asset price. For large $|x|, P(x)$ crosses over to simple exponential decay. There is however no scale invariance as previously suggested.

The more challenging task is to devise a dynamic equation that generates a time series with the same conditional averages as those of the market data. This issue was considered recently by Stolovitzky and Ching[13]. They studied a one-dimensional Langevin process defined by the following stochastic differential equation,

$$
m \frac{d^{2} x}{d t^{2}}+\gamma \frac{d x}{d t}=F(x)+\left[2 \gamma k_{B} T(x)\right]^{1 / 2} \xi(t)
$$

where $\xi(t)$ is a gaussian white noise with $\langle\xi(t)\rangle=0$ and $\left\langle\xi(t) \xi\left(t^{\prime}\right)\right\rangle=\delta\left(t-t^{\prime}\right)$. The main difference of (7) from the usual Brownian process is an $x$-dependent temperature (or noise amplitude) $T(x)$. In the over-damped limit $\gamma \rightarrow \infty$, they showed that the conditional averages are given by,

$$
\langle\ddot{x} \mid x\rangle=F(x) / m, \quad\left\langle\dot{x}^{2} \mid x\right\rangle=k_{B} T(x) / m .
$$

Combining with Eq. (4), Stolovitzky and Ching showed that the PDF in this 
limit is given by a generalised Boltzmann form,

$$
P(x)=\frac{C}{T(x)} \exp \left[\int_{0}^{x} \frac{F\left(x^{\prime}\right)}{k_{B} T\left(x^{\prime}\right)} d x^{\prime}\right] .
$$

The significance of the above result is as follows. Assuming that a given time series is generated by a Langevin process (7), one can then determine the effective force $F(x)$ and the effective temperature $T(x)$ uniquely by computing the conditional averages from the data, apart from an overall time constant. The PDF of the Langevin time series is identical to the PDF of the original data by construction.

The $\gamma \rightarrow \infty$ limit is quite suitable for the analysis of the HSI data, as we have seen from the two-point correlation function $C(\tau)$ that any memory effect about the direction of the move decays to zero rapidly. It is then suggestive to drop the inertia (i.e. mass) term in Eq. (7) altogether. Performing the scaling $t \rightarrow \gamma t$ and setting $k_{B}=1$, we can cast Eq. (7) in the form,

$$
\frac{d x}{d t}=F(x)+[2 T(x)]^{1 / 2} \xi(t)
$$

The effective force and the effective temperature are related to the conditional averages through Eq. (8). The parameter $m$ can be chosen to include a shorttime relaxation effect as seen in Fig. 1[14].

We have simulated the Langevin equation (10) using an Euler integration scheme with $\Delta t=0.1 \mathrm{~min}$. The form of the functions $F(x)$ and $T(x)$ are determined from the conditional averages (5). The PDF of the simulated minuteby-minute moves, with the same number of events as the original data, is shown in Fig. 2 (crosses). In Fig. 4 we plot both the HSI data (daily close) and the simulated index data with an artificial annual yield of $10 \%$. The gross features of the two data sets seem to be similar to the naked eye, though in the simulated data set there is no daily and weekly breaks.

One important feature which is missing in the Langevin equation (7) is the long-term volatility correlations which may last from a few days to several weeks or longer. The simulated time series has essentially the same relaxation time, on the order of a few minutes in our case, for the linear move correlation and for the volatility correlation. It is however possible to introduce volatility persistence by hand into the simulation. The effect of a nonstationary volatility on the PDF of the time series is a subject under current investigation.

Acknowledgements: We would like to thank Prof. Lam Kin at the Department of Finance and Decision Sciences, HK Baptist University for providing 


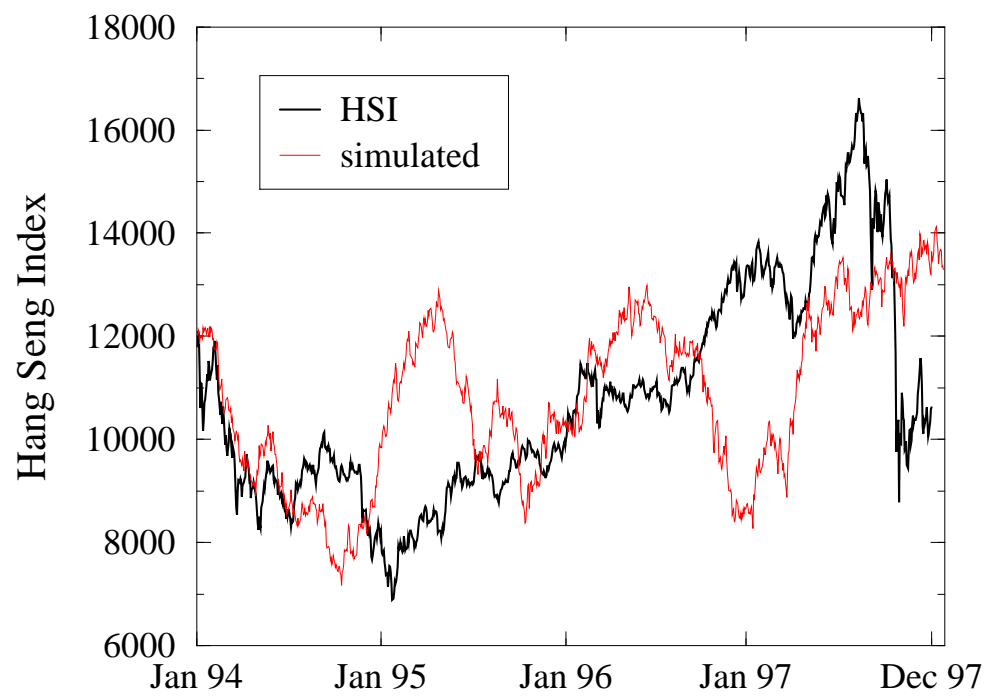

Fig. 4. Daily close of the HSI over a four year period (heavy line) and the simulated index data (dotted line).

the HSI data, and Dr. Emily Ching for sending us Ref. [13] prior to publication. The work is supported in part by the Hong Kong Baptist University under grant FRG/97-98/II-78.

\section{References}

[1] See, e.g., the electronic archive at http://netec.mcc.ac.uk and http://www.unifr.ch/econophysics.

[2] R. N. Mantegna and H. E. Stanley, Nature 376, 46 (1995).

[3] R. Mantegna and H. E. Stanley, An Introduction to Econophysics (to be published by Cambridge University Press).

[4] J.-P. Bouchaud and M. Potters, Theory of Financial Risk (to be published by Cambridge University Press).

[5] R. F. Engle, Econometrica 50, 987 (1982); see also ARCH - Selected Readings, edited by R. F. Engle (Oxford, 1995).

[6] T. Bollerslev, J. Econometrics 31, 307 (1986).

[7] B. B. Mandelbrot, J. Business Univ. Chicago 36, 394 (1963); The Fractal Geometry of Nature (W. H. Freeman and Co., New York, 1982); Physica A 263, 477 (1999), and references therein.

[8] S. Ghashghaie, W. Breymann, J. Peinke, P. Talkner and Y. Dodge, Nature 381, 767 (1996); see also A. Arneodo, J.-P. Bouchaud, R. Cont, J.-F. Muzy, M. Potters, D. Sornette, cond-mat/9607120. 
[9] R. N. Mantegna and H. E. Stanley, Physica A 239, 255 (1997); Nature 383, 587 (1996).

[10] B. Castaing, G. Gunaratne, F. Heslot, L. Kadanoff, A. Libchaber, S. Thomas, X. Z. Wu, S. Zaleski, and G. Zanetti, J. Fluid Mech. 204, 1 (1989).

[11] S. B. Pope and E. S. C. Ching, Phys. Fluids A 5, 1529 (1993); E. S. C. Ching, Phys. Rev. E 53, 5899 (1996).

[12] In Ref. [4] several classes of distributions were discussed. The closest to ours is probably the hyperbolic distribution. However, without the denominator, the PDF does not possess a sharp peak at $x=0$ which is one of the defining properties of high-frequency data.

[13] G. Stolovitzky and E. S. C. Ching, Phys. Lett. A 255, 11 (1999).

[14] The slightly under-damped behaviour seen in Fig. 1 can be simulated by including both the mass term and the friction term. Details of our study will be reported elsewhere. 